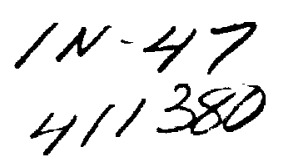

\title{
Monte Carlo Calculations of Polarized Microwave Radiation Emerging from Cloud Structures
}

by

Laura Roberti (Politecnico di Torino, Italy)

Christian Kummerow (GSFC). 0

This paper is being submitted to the Journal of Geophysical Research. It deals with basic radiative transfer theory. In particular, it presents a method for using relatively simple statistical methods (known as Monte Carlo methods) to perform 3-dimensional radiative transfer computations through arbitrarily shaped volumes. This main emphasis of this paper is on a technique to deal with non-spherical particles. Such particles, which we know to exist in raining clouds, are unique in that they are able to change the polarization state of radiation. Because of this, much more sophisticated computations than normally used are needed to predict the amount of radiation reaching a satellite. While such methods have been described in the literature, this work is unique in that the Monte Carlo method really allows the user to arbitrarily specify the size and shape of the rain cloud. Previous methods were limited to fairly simple geometric shapes such as cubes and cylinders. 


\title{
Monte Carlo Calculations of Polarized Microwave Radiation Emerging From Cloud
}

\section{Structures}

\section{Laura Roberti}

Dip. di Elettronica, Politecnico di Torino, Corso Duca degli Abruzzi 24, 10129, Torino, Italy

\begin{abstract}
Christian Kummerow
Laboratory for Atmospheres, NASA Goddard Space Flight Center, Greenbelt Maryland.
\end{abstract}

\begin{abstract}
The last decade has seen tremendous growth in cloud dynamical and microphysical models that are able to simulate storms and storm systems with very high spatial resolution, typically of the order of a few kilometers. The fairly realistic distributions of cloud and hydrometeor properties that these models generate has in turn led to a renewed interest in the three-dimensional microwave radiative transfer modeling needed to understand the effect of cloud and rainfall inhomogeneities upon microwave observations. Monte Carlo methods, and particularly backwards Monte Carlo methods have shown themselves to be very desirable due to the quick convergence of the solutions. Unfortunately, backwards Monte Carlo methods are not well suited to treat polarized radiation. This study reviews the existing Monte Carlo methods and presents a new polarized Monte Carlo radiative transfer code. The code is based on a forward scheme but uses aliasing techniques to keep the computational requirements equivalent to the backwards solution. Radiative transfer computations have been performed using a microphysical-dynamical cloud model and the results are presented together with the algorithm description.
\end{abstract}




\section{Introduction}

Passive microwave remote sensing of rainfall has always been hampered by non-linear relations between rainfall rates and satellite observed radiances. Nearly 20 years ago, Weinman and Davies [1977] and then later Kummerow and Weinman [1988,a] built radiative transfer models to account for the 3-Dimensional nature of precipitation. Unfortunately, these early models were limited to simple cuboidal geometries and much of the 3-dimensional effect was determined by the assumptions regarding the size and shape of the clouds. More recently, cloud dynamical models such as Tao et al. [1987] or Tripoli [1992] have made great strides in generating fine scale cloud and storm properties which appear quite realistic when compared to ground based radars. Because the cloud models fully specify the atmospheric structure and hydrometeor contents of the storms, these models offer the next level of realism needed to understand the radiative effects of 3-dimensional cloud and rainfall fields.

From a remote sensing point of view, the idea of using backward Monte Carlo simulations to deal with these new complex cloud structures in the microwave regime was due to Petty (1994), although that paper still dealt with abstract cloud structures. The work was quickly followed by Roberti et al., (1994), dealing explicitly with cloud dynamical model output and soon thereafter by Liu et al, (1996) who first considered polarized radiative transfer, although only in a limited sense. Backwards Monte Carlo methods were found to be very easy to implement, flexible and allowed an easy interpretation of the interactions of the radiation with the medium. Large 
absorption cross-sections meant that photons were absorbed quickly in the microwave regime leading to numerical convergence with relatively few photons. With solution speeds comparable to analytical approaches, the Monte Carlo methods are indeed attractive due to their simplicity.

One shortcoming of the backward Monte Carlo solutions is its inability to deal properly with polarization introduced by atmospheric constituents unless the vertical and horizontal extinction cross-sections are identical. Current satellite sensors such as the SSM/I and the TRMM Microwave Imager measure microwave brightness temperature $(\mathrm{Tb})$ in both the horizontal as well as the vertical polarization. Over water backgrounds, emissivities are significantly different for these polarizations and the $\mathrm{Tb}$ differences may thus be used as a measure of the total attenuation of the microwave signal as it propagates through the cloud. Land surfaces generally produce only very small polarization signatures. These signatures are almost impossible to detect in the presence of rainfall which further attenuates any surface signal. An analysis by Heymsfield, and Fulton (1994), however, revealed $85 \mathrm{GHz}$ polarization signals (TbH - TbV) of about $8-13 \mathrm{~K}$ over the stratiform precipitation region of both extra-tropical and tropical Mesoscale Convective Systems (MCS) over land. There is a similar (even if not recurring) pattern at $37 \mathrm{GHz}$ although the magnitude of the polarization signal does not appear to exceed $7 \mathrm{~K}$. No polarization signals were found in the convective portions of these storms. Heymsfield and Fulton argue that these polarization signals cannot be attributed to the surface conditions but must be attributed to atmospheric constituents and speculate that they are probably due to nonspherical hydrometeors, such as smaller ice crystals, falling slowly with an approximate horizontal orientation in the less turbulent mesoscale updraft. The lower polarisation differences 
in the convective region could be caused by the presence of randomly oriented snow particles, due to the convective motion, and to the presence of bigger, almost spherical, graupel particles.

Backward Monte Carlo schemes cannot, by design, deal with photons changing polarisation state. The main reason is that in a backward scheme the first collision is effectively the last collision in the temporal sequence. This implies that, when a photon is started, the initial polarization state is unknown. To overcome this problem, Liu et al., (1996) developed the so called "backward-forward" scheme. This scheme does allow for the treatment of polarized radiation, but requires that the extinction matrix be diagonal. Observational evidence such as the Heymsfield and Fulton study, which suggests preferentially oriented particles, cannot be dealt with using diagonal extinction matrices. The next development step for microwave radiative transfer codes must be fully polarized 3-dimensional methods. With this aim, a new forward Monte Carlo method has been developed for the solution of the radiative transfer equation to include a full treatment of the Stokes parameters.

In the process of obtaining an efficient forward Monte Carlo code, four different Monte Carlo methods have been developed and are described in the following sections: 1) a 3-D backwardforward polarized code for spherical or randomly oriented hydrometeors similar to Liu et al., (1996); 2) a direct plane parallel non polarized code; 3) a direct plane parallel polarized code for spherical or random oriented spheroidal particles; and 4) a direct plane parallel polarized code for oriented spheroidal particles. The last section examines the necessary extensions to make a fully polarized version work within a 3-dimensional framework. The aim of these codes is to simulate the radiance that could be measured by a space-borne radiometer at the top of the 
atmosphere in a specific direction needed to build a quantitative understanding of the effect of non-spherical particles upon the polarization signatures. The Goddard Cumulus Ensemble (GCE) model Tao et al. [1987] serves as a realistic hydrometeor field which can be used to study the behavior for varied hydrometeor shape and density assumptions.

\section{The cloud model and the computation of scattering parameters.}

All the radiative transfer computations in this study refer to realistic cloud profiles obtained form the cloud microphysical-dynamical model developed by Tao et al. [1987]. The cloud domain consists in $64 \times 64$ pixels at 15 minutes in the evolution of the simulated storm. A single time has been chosen which represents a mature squall line. The top boundary is considered as cosmic background with a temperature of $\mathrm{Tc}=2.7 \mathrm{~K}$, while the surface has been assumed to be Lambertian with a temperature of $300 \mathrm{~K}$ and emissivity $\varepsilon_{s}=\varepsilon_{h}=\varepsilon_{v}=0.9$. Each pixel consists of 21 vertical layers from the surface to a height of $9 \mathrm{~km}$. The cloud model specifies the height, pressure, temperature and relative humidity of each layer. Within each layer, cloud water, rain water, cloud ice, snow and graupel contents are specified. Graupel is assumed to be spherical and its density is $0.4 \mathrm{gm} \mathrm{cm}^{-3}$. Both rain and snow are assumed to be horizontally oriented oblate spheroids. The asymmetry factor for rain is the same used in Kummerow and Weinman $[1988, \mathrm{~b}]$ and its density is $1 \mathrm{gm} \mathrm{cm}^{-3}$. A Marshall and Palmer size distribution is used for snow, rain and graupel. Two different codes have been used for the computation of the scattering parameters. The first uses the T-matrix method (Barber and Hill [1990]) while the second uses the Discrete Dipole Approximation (Draine and Flatau [1988]). The codes by Barber and Hill [1990] allow the computation of the scattering matrix only for randomly oriented particles, but, 
for the considered cases, these codes were faster and more accurate of the DDA codes. It is reasonable to suppose that the polarization differences are mainly due to the different scattering and absorption coefficients and therefore, in the following simulations, the matrices for randomly oriented particles have been used. The obtained results could be slightly altered by this simplification, but the physical considerations are still valid. For spheroidal oriented particles, the extinction coefficient and the single scattering albedo have been computed both for the vertical and horizontal polarization for 30 different polar directions of incidence between $0^{\circ}$ and $90^{\circ}$. The particle symmetry allows no computations to be done for different azimuth directions.

\section{Polarized 3-D backward-forward Monte Carlo method for spherical or random oriented hydrometeors}

A backward 3-D Monte Carlo method previously developed by Roberti et al. [1994] has been extended to allow the computation of polarized radiation. Here only the necessary modifications are described. A similar Monte Carlo method has been developed by Lill et al. [1996]. In particular, here and in the following, we adopt the notation of Chandraseckar [1960] describing a beam of polarized radiation with the Stokes parameters $\mathbf{I}=\left(I_{h}, I_{v}, U, V\right)$ where $h$ and $v$ refer to two mutually orthogonal directions respectively parallel and perpendicular to the reference plane. In our case the reference plane is the $\mathrm{XY}$ plane. The photons are released at the point of each subcloud where the $T_{B}$ is to be computed, with the direction opposite to the one in which they would physically propagate. At each scattering event the position of the photon, as well as 
the incident and scattering directions are memorized. When the photon is finally absorbed, then the initial Stokes parameters are $\mathbf{I}^{0}=\left(T_{x}, T_{x}, 0,0\right)$ where $T_{x}$ is the physical temperature of the medium at the point of absorption. This assumption canbe made because the Planck function is virtually linear with temperature in the microwave regime for typical atmopheric temperatures, and can therefore be replaced by $T_{x}$. This further implies that the radiances can be interpreted as brightness temperatures rather than power per unit area. Then the path of the photon is traced forward through the scattering events and the scattering matrix is computed at each step. The Stokes vector after a scattering event is obtained from the Stokes vector before the scattering (primed variables) with the transformation:

$$
\mathbf{I}=\mathbf{L}\left(-i_{2}\right) \mathbf{T P}(\cos \theta) \mathbf{T}^{-1} \mathbf{L}\left(-i_{1}\right) / P_{11} \mathbf{I}^{\prime}=\mathbf{S I}^{\prime}
$$

where

$$
\begin{aligned}
\mathbf{L}(-i) & =\left(\begin{array}{cccc}
\cos ^{2} i & \sin ^{2} i & -1 / 2 \sin 2 \mathrm{i} & 0 \\
\sin ^{2} i & \cos ^{2} i & 1 / 2 \sin 2 \mathrm{i} & 0 \\
\sin 2 i & -\sin 2 \mathrm{i} & \cos 2 i & 0 \\
0 & 0 & 0 & 1
\end{array}\right) \\
\mathbf{T} & =\left(\begin{array}{cccc}
1 & 1 & 0 & 0 \\
1 & -1 & 0 & 0 \\
0 & 0 & 1 & 0 \\
0 & 0 & 0 & 1
\end{array}\right)
\end{aligned}
$$

and 


$$
\mathbf{P}=\left(\begin{array}{cccc}
P_{11} & P_{12} & 0 & 0 \\
P_{12} & P_{22} & 0 & 0 \\
0 & 0 & P_{33} & P_{34} \\
0 & 0 & -P_{34} & P_{44}
\end{array}\right)
$$

The definition of angles $i_{1}$ and $i_{2}$ is consistent with the one given by (Chandrasechkar [1960], pp.39) and the scattering matrix $\mathbf{P}$ coincides with the definition given by (Van de Hulst [1957], pp.44). In particular $P_{11}$ is the phase function for the unpolarized radiation. The division by $P_{11}$ in Eq. (1) is necessary to remove the bias introduced by the biased sampling of direction after scattering. In fact $\eta=\cos \vartheta$ is sampled from $P_{11}$ and $i_{1}$ is sampled randomly between 0 and $2 \pi$, which are only a first approximation of the correct distributions (Kattawar and Plass [1968], Collins et al. [1972]). The angle $i_{2}$ can computed from trigonometry (Liou [1980] pp.223). The final Stokes vector is finally derived as

$$
\mathbf{I}=\mathrm{S}_{\mathrm{N}} \ldots \mathrm{S}_{1} \mathbf{I}_{0}
$$

where $\mathrm{N}$ is the number of scattering events. The surface can be either specular or Lambertian with a given angular and polarization dependent emissivity. If an interaction with the surface occurs, a random number $r$ uniformly distributed between $[0,1]$ is compared with both the vertical and horizontal emissivities, $\varepsilon_{h}$ and $\varepsilon_{v}$. Three different events may occur. If

$$
r \leq \varepsilon_{h}, r \leq \varepsilon_{v}
$$


then the photon is absorbed and the corresponding Stokes vector is $\mathbf{I}_{0}=\left(T_{s}, T_{s}, 0,0\right)$, where $T_{s}$ is the surface temperature. If

$$
r>\varepsilon_{h}, r>\varepsilon_{\nu}
$$

then the photon is scattered, else if

$$
r \leq \varepsilon_{h}, r>\varepsilon_{v}
$$

then the photon is scattered and traced through the successive scattering events until it is finally absorbed with $\mathbf{I}_{0}=\left(0, T_{x}, 0,0\right)$. Then the scattering events are traced forward and the Stokes vector is multiplied by the appropriate scattering matrices as in Eq. (1), until the interaction with the surface occurs. At that point the surface temperature $T_{S}$ is added to the $I_{h}$ component of the current Stokes vector. If the surface is Lambertian the scattering matrix is given by

$$
\mathbf{T P}(\cos \theta) \mathbf{T}^{-1}=\frac{\cos \theta}{\pi}\left(\begin{array}{cccc}
0.5 & 0.5 & 0 & 0 \\
0.5 & 0.5 & 0 & 0 \\
0 & 0 & 0 & 0 \\
0 & 0 & 0 & 0
\end{array}\right)
$$

An analog procedure is followed if $r>\varepsilon_{h}, r \leq \varepsilon_{\nu}$. To test the validity of the code, the results have been compared with those in Weinman and Guetter [1977] for a plane parallel cloud and they have been found in good agreement. This code can successfully take into account spherical 
or randomly oriented spheroidal particles, in which cases the extinction and scattering parameters are equal for the two polarization. Only the surface emissivity can be different for the two polarization. The code can take into account horizontal as well as vertical inhomogeneities of the medium.

\section{Non polarized direct Monte Carlo method}

The inability of backward MC, or even backward-forward MC methods to deal with nondiagonal scattering matrices requires the development of a polarized forward $\mathrm{MC}$ method. The first step was to develop a non-polarized forward Monte Carlo code competitive in terms of speed of convergence with the backward Monte Carlo code developed by Roberti et al. [1994]. For this and the following codes the medium is considered to be plane parallel, even if the method could be extended to consider the horizontal inhomogeneities of the cloud as suggested in Sec.6.3. With a forward Monte Carlo method the photons are released by the medium coherently with the source parameters and their propagation is followed until the escape from the medium. Unfortunately, to simulate the $T_{B}$ that could be measured by a space-borne radiometer, only the photons which escape the medium in a specific point and direction will be taken into account. This usually involves high computational times because only a fraction of the photons contribute to the radiance. To overcome this problem a number of biasing techniques (see also Roberti [1997]) are used.

\subsection{Source parameters}


The first problem in the definition of the source concerns the choice between the atmosphere, the surface, or the cosmic background as an emission source and has been solved by computing the irradiance which is proportional to the number of photons emitted. The total irradiance for a plane parallel atmosphere of height $Z_{0}$ is equal to

$$
F_{a}=\int_{0}^{z_{0}} \int_{4 \pi} \varepsilon(z) T(z) k(z) d \Omega d z=4 \pi \int_{0}^{z_{0}} \varepsilon(z) T(z) k(z) d z
$$

where $\varepsilon, T, k$ are respectively the emissivity, the physical temperature and the extinction coefficient at height $z$. The irradiance of a Lambertian surface is equal to

$$
F_{s}=\int_{2 \pi} \varepsilon_{s} T_{s} \cos \theta d \Omega=\pi \varepsilon_{s} T_{s}
$$

where $\varepsilon_{S}$ and $T_{S}$ represent respectively the surface emissivity and temperature. The irradiance of the cosmic background of temperature $T_{c}=2.7 \mathrm{~K}$ is equal to

$$
F_{c}=\pi T_{c}
$$

The photon is released from the atmosphere if 


$$
r<\frac{F_{a}}{F_{a}+F_{s}+F_{c}}
$$

and in a similar manner for the other sources. In the atmosphere a uniform emission in the vertical direction has been chosen. The direction of emission will be isotropic for the atmosphere and Lambertian for the surface.

\subsection{Tracing procedure}

The distance to collision is computed using two different methods depending on whether the photon is downwelling or upwelling. If the photon extended path intersects the surface, the optical distance to collision $\tau_{\text {coll }}$ is computed as

$$
\tau_{\text {coll }}=-\ln (r)
$$

where $r$ is a random number uniformly distributed between [0,1]. If the photon extended path intersects the upper boundary of the atmosphere, a collision is forced before the photon escapes the medium by selecting the optical distance from the truncated exponential distribution Collins et al [1982] so that

$$
\tau_{\text {coll }}=-\ln \left[1-r\left(1-e^{-\tau_{i}}\right)\right]
$$


where $\tau_{i}$ is the optical distance along the photon path, from the current photon position to the upper boundary of the atmosphere. In this case the photon weight is adjusted multiplying it by the factor

$$
\left(1-e^{-\tau_{i}}\right)
$$

After computing the distance to collision the photon can find itself in two possible situations. 1) If the photon crosses a boundary before interacting with the medium it is advanced to the boundary and a new distance to collision is computed taking into consideration the distance already traveled House and Avery [1969]. The photon is then relaunched from the boundary it would have crossed.

2) If a collision occurs, then a scattering event is forced, thus avoiding absorption. The corresponding bias is removed by multiplying the photon weight by the single scattering albedo $A(z)$ of the medium in the current position of the photon, which represents the probability of photon survival. If the collision is with the surface the photon weight is multiplied by $\left(1-\varepsilon_{s}\right)$. The new direction after scattering is computed using the phase function. Furthermore, to reduce computational times, the contribution that the photon would given to the $T_{B}$ if scattered in the viewing direction and if it would propagate to the upper boundary of the atmosphere without any further interaction with the medium is computed and the photon weight is modified accordingly. In the following $\mathrm{W}_{\mathrm{i}, \mathrm{n}}$ denotes the weight effectively attached to the $\mathrm{n}$-th photon during the propagation at the $i$-th scattering event and $\mathrm{W}^{\prime}{ }_{i, n}$ indicates the weight attached to the $\mathrm{n}$-th photon when its contribution to the $T_{B}$ if scattered (or emitted) in the viewing direction is computed. The weights are modified as follows 


$$
\begin{array}{ll}
\mathrm{W}_{0, \mathrm{n}}=1(\text { emission }) & \mathrm{W}_{0, \mathrm{n}}^{\prime}=p\left(\cos \Theta_{0}\right) \mathrm{e}^{-\tau_{0}} \mathrm{~W}_{0, \mathrm{n}} \\
\mathrm{W}_{\mathrm{t}, \mathrm{n}}=\Lambda_{1} \mathrm{~W}_{0, \mathrm{n}} & \mathrm{W}_{1, \mathrm{n}}^{\prime}=p\left(\cos \Theta_{1}\right) \mathrm{e}^{-\tau_{1}} \mathrm{~W}_{1, \mathrm{n}} \\
\mathrm{W}_{2, \mathrm{n}}=\Lambda_{2} \mathrm{~W}_{1, \mathrm{n}} & \mathrm{W}_{2, \mathrm{n}}^{\prime}=p\left(\cos \Theta_{2}\right) \mathrm{e}^{-\tau_{2}} \mathrm{~W}_{2, \mathrm{n}} \\
\mathrm{W}_{i-1, \mathrm{n}}=\Lambda_{i+1} \mathrm{~W}_{i, n} & \mathrm{~W}_{i+1, \mathrm{n}}^{\prime}=p\left(\cos \Theta_{i+1}\right) \mathrm{e}^{-\tau_{i, 1}} \mathrm{~W}_{i+1, \mathrm{n}}
\end{array}
$$

where $\Lambda_{i}$ is the single scattering albedo at the considered layer, $p\left(\cos \Theta_{i}\right)$ is either the atmospheric or the surface phase function evaluated on the angle between the incidence and the viewing direction, $\tau_{i}$ is the optical depth from the point in which the $i$-th collision has occurred to the upper boundary along the viewing direction. The phase function $p\left(\cos \Theta_{0}\right)$ for the emission contribution is $1 / 4 \pi$ for the atmosphere and $\cos \left(\theta_{v}\right) / \pi$ for the Lambertian surface where $\theta_{v}$ is the polar viewing angle. The photon continues to propagate until the associated weight becomes less than the threshold below which the photon is eliminated. The threshold value has been fixed at $10 \mathrm{E}-6$ in all the computations that follow.

\section{$4.3 \mathrm{~T}_{\mathrm{B}}$ computation}

The irradiance emitted by the atmosphere is computed as the sum of the contributions of all the layers of infinitesimal thickness $d z$ (Eq.(10)). With a Monte Carlo method, the integral in Eq.(10) is computed numerically as 


$$
F_{a}=4 \pi \int_{0}^{z_{0}} \varepsilon(z) T(z) k(z) d z \approx 4 \pi \frac{Z_{0}}{N_{n}} \sum_{n=1, N_{a}} \varepsilon_{n} T_{n} k
$$

where $N_{a}$ is the number of photons emitted by the atmosphere and $Z_{0}$ is the atmosphere height. $\varepsilon_{n}, T_{n}, k_{n}$ depend on the height of emission $z$ of the $n$-th photon. The fraction $\widetilde{F}_{a}$ of $F_{a}$ which effectively contributes to the $T_{B}$ is

$$
\widetilde{F}_{a}=4 \pi \frac{Z_{0}}{N_{n}} \sum_{n=1, N_{a}} \varepsilon_{n} T_{n} k_{n} \sum_{i=0, N}\left(\prod_{j=1, i} \Lambda_{j}\right) p\left(\cos \Theta_{i}\right) e^{-\tau_{i}} d \Omega_{v i}=4 \pi \frac{Z_{0}}{N_{a}} \sum_{n=1, N_{a}} \varepsilon_{n} T_{n} k_{n} \sum_{i=0, N} W_{i, n}^{\prime} d \Omega_{v i}
$$

where $N$ is the number of scattering events of the considered photon ( $i=0$ indicates emission), $p(\cos \Theta) \mathrm{e}^{-\tau} d \Omega_{v}$ represents the probability that the photon after a scattering event propagates to the upper boundary of the medium in the viewing direction (i.e. in an infinitesimal solid angle $d \Omega_{v}$ ) without undergoing any other collision. The atmospheric contribution to the $\mathrm{T}_{\mathrm{B}}$ can be computed by dividing $\widetilde{F}_{a}$ by $\cos \left(\theta_{v}\right) d \Omega_{\mathcal{V}}$.

$$
T_{B a} \approx \frac{4 \pi}{\cos \theta_{v}} \frac{Z_{0}}{N_{a}} \sum_{n=1, N_{a}} \varepsilon_{n} T_{n} k_{n} \sum_{i=0, N} W_{i, n}^{\prime}
$$

The contribution to the $T_{B}$ given by the surface emission is 


$$
T_{B S} \approx \frac{\pi}{\cos \theta_{v}} \frac{Z_{0}}{N_{s}} \sum_{n=1, N s} \varepsilon_{s} T_{s} \sum_{i=0, N} W_{i, n}^{\prime}
$$

where $N_{S}$ is the number of photons emitted by the surface. The contribution to the $\mathrm{T}_{\mathrm{B}}$ given by the cosmic background is

$$
T_{B c} \approx \frac{\pi}{\cos \theta_{v}} \frac{Z_{0}}{N_{c}} \sum_{n=1, N_{c}} T_{c} \sum_{i=0, N} W_{i, n}^{\prime}
$$

where $N_{C}$ is the number of photons emitted by the cosmic background. Consequently the $\mathrm{T}_{\mathrm{B}}$ can be computed as a sum of Eqs. (20), (21) and (22).

$$
T_{B}=T_{B a}+T_{B s}+T_{B c}
$$

The results of this code have been compared with those of an equivalent backward code [Roberti et al., 1995]. In Fig.1 the resulting $T_{B}$ are presented both for a subcloud in the anvil (stratiform region) and a subcloud in the core (convective region) for 3 different frequencies. The convergence of the forward code (dashed line) is slower and the oscillations are higher. Nevertheless, in the worst case, only 500000 photons are needed to keep the oscillations below IK. 


\section{Direct polarized Monte Carlo method (spherical or random oriented spheroidal hydrometeors)}

The code described above has been extended to include the polarization to treat spherical or random oriented spheroidal hydrometeors.

\subsection{Source Parameters}

The choice of the photons emission source is based, as for the unpolarized radiation, on the irradiances of the different sources. For spherical or random oriented spheroidal particles the extinction matrix for the Stokes vector is diagonal $K=\operatorname{diag}(k)$ which means that the four components of the Stokes vector are subject to the same extinction. Therefore the distance to collision can be computed as for the unpolarized radiation.

\subsection{The tracing procedure}

After emission, a scattering event is forced at every collision by multiplying the photon weight by the single scattering albedo and the new direction is determined by sampling the phase function. The new Stokes vector after a collision is computed as in Eq. (1). As explained in Sec. 4.2 a drastic reduction of computational times is obtained by considering the contribution to the final Stokes vector given by each photon collision. The photon weight of the n-th photon is modified as follows: 


$$
\begin{array}{cl}
\mathbf{W}_{0, n}=\mathbf{1} & \mathbf{W}_{0, n}^{\prime}=p\left(\cos \Theta_{0}\right) \mathrm{e}^{-t_{0}} \mathbf{W}_{0, n} \\
\mathbf{W}_{1, n}=\widetilde{\mathbf{S}}_{1} \mathbf{L}_{1} \quad \mathbf{W}_{0, n} & \mathbf{W}_{\mathrm{l}, n}^{\prime}=\mathrm{e}^{-\tau_{1}} \mathbf{S}_{1} \mathbf{L}_{1} \mathbf{W}_{0, n} \\
\mathbf{W}_{2, n}=\widetilde{\mathbf{S}}_{2} \mathbf{L}_{2} \mathbf{W}_{1, n} & \mathbf{W}_{2, n}^{\prime}=\mathrm{e}^{-\tau_{2}} \mathbf{S}_{2} \mathbf{L}_{2} \quad \mathbf{W}_{1, n} \\
\mathbf{W}_{i+1, n}=\widetilde{\mathbf{S}}_{i+n} \mathbf{L}_{i+1} \mathbf{W}_{i, n} & \mathbf{W}_{i+1, n}^{\prime}=\mathrm{e}^{-\tau_{i+1}} \mathbf{S}_{i+1} \mathbf{L}_{i+1} \mathbf{W}_{i, n}
\end{array}
$$

The matrices $\widetilde{\mathbf{S}}$ in Eq.(24) are evaluated on the angle between the photon incidence and the scattering direction, while the matrices $\mathbf{S}$ are evaluated between the incidence and the viewing direction. The expression for matrices $\mathbf{S}$ is given in Eq.(1) and

$$
\mathbf{L}=\operatorname{diag}\left(\Lambda_{h}, \Lambda_{v}, \frac{\Lambda_{h}+\Lambda_{v}}{2}, \frac{\Lambda_{h}+\Lambda_{v}}{2}\right)
$$

For spherical or random oriented aspherical hydrometeors the single scattering albedos for the horizontal and vertical polarization $\Lambda_{h}, \Lambda_{v}$ are equal. The photon continues the propagation until the first two components of the Stokes vector become lower of a fixed threshold and the photon is eliminated.

\subsection{Computation of the Stokes vector}

As for the unpolarized radiation the contribution to the final Stokes vector given by photons emitted respectively by the atmosphere and the surface (cosmic background) is respectively 


$$
\begin{gathered}
\mathbf{I}_{a} \approx \frac{4 \pi}{\cos \theta_{v}} \frac{Z_{0}}{N_{a}} \sum_{n=1, N_{n}} \sum_{i=0 N} \mathbf{W}_{i, n}^{\prime}\left(\begin{array}{c}
k_{n} \varepsilon_{n} T_{n} \\
k_{n} \varepsilon_{n} T_{n} \\
0 \\
0
\end{array}\right) \\
\mathbf{I}_{S(c)} \approx \frac{4 \pi}{\cos \theta_{v} N_{S(c)}} \sum_{n=1, N_{S(c)}} \sum_{i=0, N} \mathbf{W}_{i, n}^{\prime}\left(\begin{array}{c}
\varepsilon_{S} T_{S}\left(T_{c}\right) \\
\varepsilon_{S} T_{S}\left(T_{c}\right) \\
0 \\
0
\end{array}\right)
\end{gathered}
$$

The Stokes vector at any computational point and direction is given by

$$
\mathbf{I}=\mathbf{I}_{a}+\mathbf{I}_{s}+\mathbf{I}_{c}
$$

Numerical results will be presented in Sec.6.4.

\section{Direct polarized Monte Carlo method for oriented spheroidal particles.}

Applying a backward or even a backward forward Monte Carlo scheme when oriented spheroidal particles are involved gives rise to a number of problems. The angular dependence of the single scattering albedos is such that the probability of scattering is different depending on the photon propagating towards or from the detector. The use of a direct method seems ideal to avoid such problems. Furthermore the extinction matrix is of the type 


$$
\mathbf{K}_{\mathrm{e}}(x, y, z, \theta)=\left(\begin{array}{cccc}
k_{h} & 0 & 0 & 0 \\
0 & k_{v} & 0 & 0 \\
0 & 0 & \frac{k_{h}+k_{v}}{2} & k_{c} \\
0 & 0 & -k_{c} & \frac{k_{h}+k_{v}}{2}
\end{array}\right)
$$

and therefore a different extinction coefficient is associated to each Stokes component and this must be carefully considered in the photon propagation. Since the matrix is not diagonal, the extinction of each Stokes component depend also on the values of other components. A backward method seems to be useless in this respect because the Stokes vector is unknown during the propagation.

\subsection{Source parameters}

The choice of the emission source is made in a way similar to the ones previously described. For the atmosphere the following quantities are computed, which represent the average number of photons emitted by the atmosphere and the surface:

$$
\begin{aligned}
& F_{a}=\frac{F_{a_{h}}+F_{a_{v}}}{2} \\
& F_{s}=\frac{F_{s_{h}}+F_{s_{v}}}{2}
\end{aligned}
$$

$F_{a_{h}}, F_{a_{v}}, F_{s_{i b}}, F_{S_{v}}$ are the same quantities as in Eqs.(10-11) except that the emissivities can be different for the horizontal and vertical polarization. Eq.(12) is still valid for the cosmic 
background. These quantities are necessary for the computation of the unknown Stokes vector. To increase the speed of convergence, it is useful to employ quantities which represent the real proportion between the energy emitted by the different sources. In the following the extinction matrix will be considered as diagonal, neglecting the circular polarization term. It is in fact possible to assume, in the case of thermal emission, that $U$ and $V$ are relatively small with respect to $I_{h}$ and $I_{v}$. To overcome the problem of different extinction coefficients for the different components of the Stokes vector, another biasing techniques is introduced sampling the distance to collision from a biased probability distribution and multiplying the Stokes vector by proper coefficients to remove the statistical perturbation introduced. For downwelling photons the biased probability density for the optical distance to collision is

$$
\widetilde{p}(\tau) d \tau=\mathrm{e}^{-\widetilde{k} x} d(\widetilde{k} x)
$$

where $\widetilde{k}$ is an extinction coefficient computed as

$$
\tilde{k}=\frac{1}{\log \left(\frac{2}{\mathrm{e}^{-1 / k_{\mathrm{h}}}+\mathrm{e}^{-1 / k_{\mathrm{v}}}}\right)}
$$

where $k_{h}$ and $k_{v}$ are the extinction coefficients for the two polarizations. This choice has been made in order to minimize the multiplying factors for the elimination of the bias. The distance to collision is given by 


$$
d_{\text {coll }}=-\frac{1}{\widetilde{k}} \log (r)
$$

and the matrix for the correction of the bias is given by

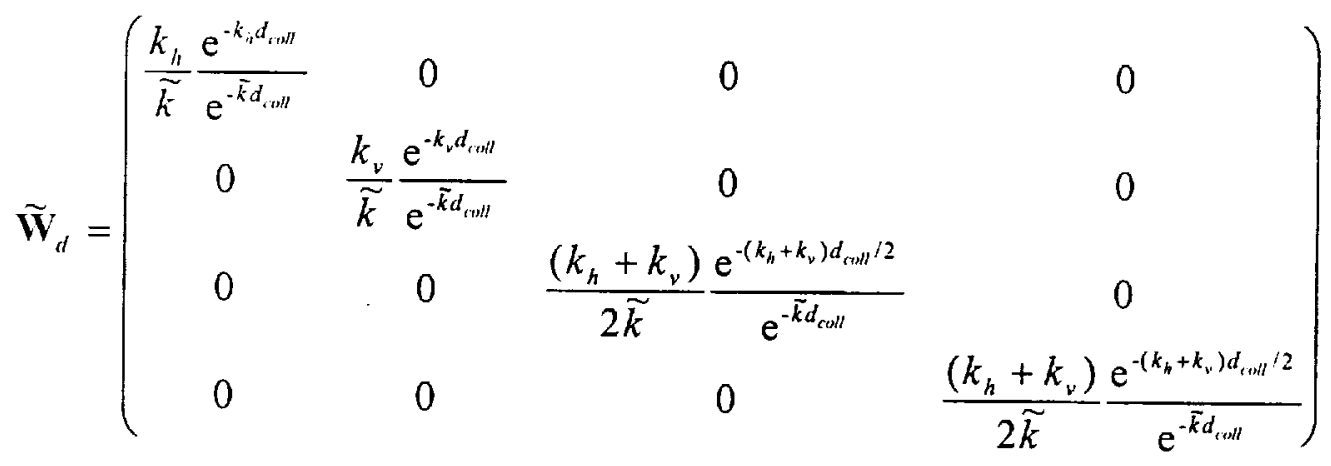

For upwelling photons, as explained in Sec. 4.2, a collision is forced before photons escape the medium and the distance to collision becomes

$$
d_{\text {coll }}=-\frac{1}{\widetilde{k}} \log \left[1-r\left(1-\mathrm{e}^{-\tilde{\tau}}\right)\right]
$$

where $\widetilde{\tau}$ is the optical thickness computed from the current photon position to the top of the atmosphere in the direction of propagation using the extinction coefficient $\widetilde{k}$. The weight correction matrix is similar to the one in Eq.(35), except that each element is still multiplied by a factor $\left(1-\mathrm{e}^{-\tilde{r}}\right)$. Some attention must be paid to the computation of the new distance to collision after the crossing of an internal boundary of the medium. The true distance to collision probability densities for a photon crossing the boundary between layer 1 and 2 are 


$$
\begin{array}{cc}
p_{h}(\tau) d \tau=\mathrm{e}^{-k_{11} d_{1}} \mathrm{e}^{-k_{h 2}\left(d-d_{1}\right)} d\left(k_{h 2} d\right) & d>d_{1} \\
p_{v}(\tau) d \tau=\mathrm{e}^{-k_{v 1} d_{1}} \mathrm{e}^{-k_{v 2}\left(d-d_{1}\right)} d\left(k_{v 2} d\right) & d>d_{1}
\end{array}
$$

for the $\mathrm{H}$ and $\mathrm{V}$ polarized photons, where $d_{1}$ is the distance between the current photon position to the boundary in the direction of propagation of the photon. The biased probability density with which the distance to collision inside the new layer is corrected taking into account the distance already travelled is

$$
\widetilde{p}(\tau) d \tau=\mathrm{e}^{-\tilde{k}_{1} d_{1}} \mathrm{e}^{-\tilde{k}_{2}\left(d-d_{1}\right)} d\left(\tilde{k}_{2} d\right) \quad d>d_{1}
$$

The distance to collision in the second medium becomes

$$
d_{\text {coll-new }}=\frac{\widetilde{k}_{1}}{\widetilde{k}_{2}}\left(d_{\text {coll-old }}-d_{1}\right)
$$

where $d_{\text {coll-old }}$ is the distance to collision computed in the first medium. Therefore the weight correction matrix is a diagonal matrix with elements 


$$
\begin{aligned}
& \widetilde{\mathbf{W}}_{\mathrm{II}}=C \frac{k_{h 2}}{\widetilde{k}_{2}} \frac{\mathrm{e}^{-k_{h_{1}} d_{1}} \mathrm{e}^{-k_{i 2} d_{\text {colll-new }}}}{\mathrm{e}^{-\widetilde{k}_{1} d_{1}} \mathrm{e}^{-\widetilde{k}_{2} d_{\text {toll-nww }}}}
\end{aligned}
$$

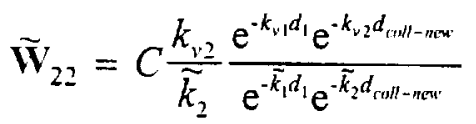

$$
\begin{aligned}
& \widetilde{\mathbf{W}}_{33}=C \frac{k_{h 2}+k_{v 2}}{2 \widetilde{k}_{2}} \frac{\mathrm{e}^{-\frac{k_{h 1}+k_{v 1}}{2} d_{1}} \mathrm{e}^{-\frac{k_{h 2}+k_{v 2}}{2} d_{\text {coth - new }}}}{\mathrm{e}^{-\widetilde{k}_{1} d_{1}} \mathrm{e}^{-\widetilde{k}_{2} d_{\text {oull-new }}}}
\end{aligned}
$$

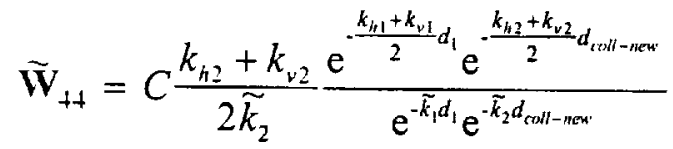

$C$ is equal to $\left(1-\mathrm{e}^{-\bar{f}}\right)$ for upwelling photons and $C=1$ in the opposite case.

\subsection{The tracing procedure}

The photon weights during the propagation are modified as follows

$$
\begin{array}{ll}
\boldsymbol{W}_{0}=\overrightarrow{\mathbf{1}} & \boldsymbol{W}_{0}^{\prime}=p\left(\cos \Theta_{0}\right) \mathbf{E}_{0} \boldsymbol{W}_{0} \\
\boldsymbol{W}_{1}=\widetilde{\mathbf{S}}_{1} \mathbf{K}^{-1}\left(z_{1,}, \theta_{0}\right) \mathbf{K}\left(z_{1}, \theta_{1}\right) \mathbf{L}\left(z_{1}, \theta_{0}\right) & \boldsymbol{W}_{1}^{\prime}=\mathbf{S}_{1} \mathbf{K}^{-1}\left(z_{1}, \theta_{0}\right) \mathbf{K}\left(z_{1}, \theta_{o s}\right) \mathbf{E}_{1} \mathbf{W}_{0} \\
\boldsymbol{W}_{2}=\widetilde{\mathbf{S}}_{2} \mathbf{K}^{-1}\left(z_{2,}, \theta_{1}\right) \mathbf{K}\left(z_{2}, \theta_{2}\right) \mathbf{L}\left(z_{2}, \theta_{1}\right) \boldsymbol{W}_{1} & \boldsymbol{W}_{2}^{\prime}=\mathbf{S}_{2} \mathbf{K}^{-1}\left(z_{2,}, \theta_{1}\right) \mathbf{K}\left(z_{2,}, \theta_{o s}\right) \mathbf{E}_{2} \mathbf{W}_{1} \\
\boldsymbol{W}_{i-1}=\widetilde{\mathbf{S}}_{i} \mathbf{K}^{-1}\left(z_{i+1}, \theta_{i}\right) \mathbf{K}\left(z_{i+1}, \theta_{i+1}\right) \mathbf{L}\left(z_{i+1}, \theta_{i}\right) W_{i} & W_{i+1}{ }^{\prime}=\mathbf{S}_{i+1} \mathbf{K}^{-1}\left(z_{i+1}, \theta_{i}\right) \mathbf{K}\left(z_{i+1}, \theta_{o s}\right) \mathbf{E}_{i+1} \mathbf{W}_{i}
\end{array}
$$

where 


$$
\mathbf{E}_{i}=\operatorname{diag}\left(\mathrm{e}^{-\tau_{\mathrm{L}, i}}, \mathrm{e}^{-\tau_{\mathrm{v}, \mathrm{i}}}, \mathrm{e}^{-\tau_{\mathrm{U}, \mathrm{i}}}, \mathrm{e}^{-\tau_{\mathrm{V}, \mathrm{i}}}\right)
$$

and $\tau_{(h, v, U, V), i}$ are the optical thickness from the $i$-th scattering point to the observation point computed with the appropriate extinction coefficient

$$
\mathbf{K}(z, \theta)=\operatorname{dicug}\left(k_{h}(z, \theta), k_{v}(z, \theta), \frac{k_{h}(z, \theta)+k_{v}(z, \theta)}{2}, \frac{k_{h}(z, \theta)+k_{v}(z, \theta)}{2}\right)
$$

The multiplication by $\mathrm{K}^{-1}$ in Eqs. (41) is introduced to take into account the angular dependency of the extinction coefficient. Matrix L is Eq.(25). The final Stokes vector is computed with formulae analogous to Eqs.(26-28).

\subsection{Extension of the code to the 3-D case}

The main problem in the extension of the forward codes to the 3-D case deals with the treatment of the emission sources. If the horizontal inhomogeneities of the medium are to be taken into account photons should be released by the different layers of the different subclouds according to the thermal contribution. For each subcloud $n x, n y$ a threshold must be fixed as (see Eqs. $10,11,12)$

$$
\operatorname{TrS}_{n x, n y}=F_{a_{n x, m y}}+F_{s_{n, w y}}+F_{c_{u x, y, y}}
$$


If $N X C L, N Y C L$ are the subcloud numbers in the $x$ and $y$ directions, a photon is emitted by subcloud $n x, n y$ if a random number $r$ uniformly distributed between $[0,1]$ satisfies

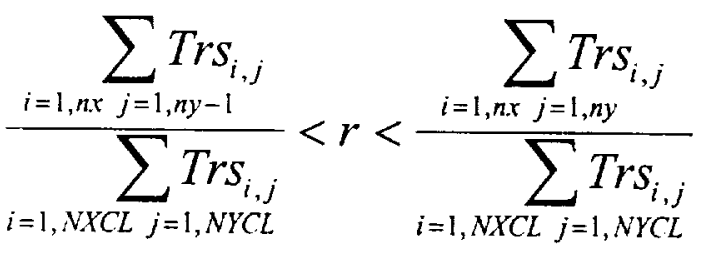

Once the subcloud has been determined, the vertical position for emission inside each subcloud can be prescribed as in Sec. 4.1. If the subcloud horizontal dimension is small compared to the vertical dimension, the photons are simply started at the center or the subcloud. Emission points must be sampled uniformly in the case that the subcloud dimension is large compared to the vertical dimension. The computation of the Stokes vector must be partially modified since the Stokes vector must be computed at the top of each subcloud. At each scattering event a line must be ideally drawn from the point of collision to the top of the cloud in the viewing direction. The line will intersect the top of only one of the subclouds. The scattering event will give a contribution only to the Stokes vector associated with that subcloud and the contribution can be computed as described in Sec. 4.3 for the unpolarized case. The rest of the algorithm remains unchanged. Photons crossing the vertical boundary between two subclouds or the horizontal boundary between two layers are treated analogously to the plane parallel methods.

\subsection{Numerical Results}


The convergence of the two forward polarized codes has been analyzed with reference to the backward-forward code. The resulting $T_{H}$ and $\Delta T_{V-H}$ are presented for a subcloud in the stratiform region (Fig. 2) for 3 different frequencies. Similar results can be obtained for a subcloud in the convective region. A Lambertian surface with emissivities $\varepsilon_{s}=\varepsilon_{h}=\varepsilon_{v}=0.9$ has been chosen in order to analyze the polarization signature introduced by atmospheric scattering. Considering spherical hydrometeors the resulting $\Delta T_{V-H}$ are negligible. The same considerations done for the forward unpolarized code are still valid here. The convergence of the forward codes is slower, but, if the required accuracy is of the order of $1 \mathrm{~K}$, an average number of 500000 photons is sufficient.

To simulate the observed polarized results (Heymsfield and Fulton [1994]), a cross section of the cloud model, made of 64 subclouds, normal to the main convective line is considered. A sensitivity analysis of the $\Delta T_{V-H}$ as a function of snow density, asymmetry factor ( $\left.a / b\right)$ and concentration using plane parallel computations on single subclouds is performed. In order to avoid changing the total ice concentration, snow concentration is varied by reducing graupel and keeping the total ice mass constant. The results are presented for a subcloud in the stratiform region (Fig. 3) and one in the convective region (Fig. 4). The resulting $\Delta T_{V-H}$ are shown, as a function of the asymmetry factor, for density of snow equal to 0.1 and $0.2 \mathrm{~g} / \mathrm{m}^{3}, 5$ different percentages of graupel transformed to snow $(\mathrm{Perc}=0,0.25,0.50,0.75$ and 1.00$)$ and at 3 frequencies $(19,37$ and $85 \mathrm{GHz})$. In particular the concentrations of snow and graupel have been computed as 


$$
\begin{aligned}
& \text { snow }=\text { snow }+ \text { graupel } * \text { Perc } \\
& \text { graupel }=\text { graupel } *(1-\text { Perc })
\end{aligned}
$$

The general behavior of the plots is the one expected: the $\Delta T_{V-H}$ increase at increasing density and percentage of graupel transformed to snow. Furthermore the $\Delta T_{V-H}$ are higher when the asymmetry factor is higher, and this appears more clearly when the percentage of graupel transformed to snow is more than $50 \%$. If the snow content is low (Perc=0, 0.25), the $\Delta T_{V-H}$ at lower frequencies are higher than those at $85 \mathrm{GHz}$. This might be due to the fact that the radiation at $85 \mathrm{GHz}$ is more sensitive to the depolarizing effect of graupel scattering. To obtain the desired $\Delta T_{V-H}$ in the anvil the content of snow originally present in the cloud seems to be too low. It is necessary to transform $50 \%$ of the graupel to snow to start obtaining a $\Delta T_{V_{-H}}$ of $11 \mathrm{~K}$ at $85 \mathrm{GHz}$. On the other hand, if all the graupel is converted to snow, the $\Delta T_{V-H}$ become too high. In the core of the cloud the increase of snow content does not give $\Delta T_{V-H}$ similar to the observed ones. For Perc $<0.5$ the influence of the remaining graupel is still too high, maintaining the resulting $\Delta T_{V-H}$ relatively low. On the other hand, for high content of oriented spheroidal snow particles ( $\operatorname{Perc}>0.75$ ), the $\Delta T_{V-H}$ become too high at all frequencies, especially for high values of the asymmetry factor. These considerations seems to confirm the hypothesis of the presence of bigger spheroidal or randomly oriented ice particles in the core of storm, due to the updraft motion. 
Since the percentage of graupel that, transformed to snow, gives $\Delta T_{V-H}$ in accordance with the observed results varies with the different subclouds, we tried to transform graupel to snow, starting from the higher layers of each of the 64 subclouds, for a total of $1.5 \mathrm{~kg} / \mathrm{m}^{2}$. The resulting $T_{H}$ and $\Delta T_{r_{-H}}$ are shown at 19,37 and $85 \mathrm{GHz}$ in Fig.5A, B respectively. The density of snow is $0.1 \mathrm{~g} / \mathrm{cm}^{3}$, the asymmetry parameter is 0.3 .

\section{Conclusions}

Four different Monte Carlo codes for the computation of microwave radiation emerging from cloud structures have been presented. Three codes allow to take into account polarization. One of them is 3-D, but treats only spherical or randomly oriented hydrometeors which give only small polarization signal. The code which can deal with oriented spheroidal particles is currently plane parallel. Future work will concentrate on the extension of this code to the $3-\mathrm{D}$ case. The convergence and accuracy of the forward Monte Carlo codes is lower with respect to backward codes, but computational times are limited thanks to the biasing techniques that have been used. Nevertheless Forward Monte Carlo codes they seem to be the only way to treat polarization when oriented aspherical particles are involved.

One of the forward Monte Carlo codes has been used for a sensitivity analysis of the $\Delta T_{V-H}$ as a function of snow parameters. To summarize the results it is possible to say that, while many combinations of density and asymmetry factor can explain the observed $\Delta T_{V-H}$, it was found that converting a total of $1.5 \mathrm{Kg} / \mathrm{m}^{2}$ of graupel into snow throughout the cloud model simulation produced generally adequate agreement with observations. The results represent a 
contribution to the understanding of clouds microphysics in relation to radiometer observables at millimeter frequencies. While the poor knowledge of the real cloud parameters is underlined, the effort is toward creating a foundation for realistic cloud modeling.

\section{Acknowledgments}

The authors thank Dr. W.-K. Tao for providing the three-dimensional micropysical cloud model. Laura Roberti acknowledges partial financial support from the Euro TRMM Project. She would like to further acknowledge the support from C.N.R. I.F.A., Rome.

\section{References}

Barber P.W., and S.C.Hill, Light Scattering by Particles: Computational Methods, (World Scientific, Singapore, 1990).

Chandrasekhar S., Radiative Transfer, (Dover Publications, New York, 1960, pp.39).

Collins D.G., W. G. Blattner, M. B. Wells, and H.G. Horak, "Backward Monte Carlo calculations of the polarization characteristics of the radiation emerging from spherical-shell atmospheres," Appl. Opt., 11, 2684-2696, 1972.

Draine B.T. and P.J. Flatau, "Discrete-dipole approximation for scattering calculations, Astrophys". J., 333, 848-872, 1988. 
Haferman J.L., Smith T.F. and Krajewski W.F., “A multi-dimensional discrete-ordinates method for polarized radiative transfer. Part I: validation for randomly oriented axisymmetric particles", J. Quant. Spectrosc. Radiat. Transfer, 58, No. 3, 379-398, 1997.

Heymsfield G.M., and R.Fulton, "Passive Microwave and Infrared Structure of Mesoscale Convective Systems", Meteorol. Atmos. Phys., 54, 123-139, 1994.

House L.L. and L.W. Avery, "The Monte Carlo technique applied to radiative transfer", J. Quant. Spectr. Radiat. Transfer, 9, 1579-1591, 1969.

Kummerow, C. and J. A. Weinman, "Determining microwave brightness temperatures from precipitating horizontally finite and vertically structured clouds," J. Geophys. Res., 93, 37203728,1988 ,a.

Kummerow C., and J.A. Weinman, "Radiative Properties of Deformed Hydrometeors for Commonly Used Passive Microwave Frequencies," IEEE TGRS, vol.26, 629-638, 1988,b.

Liou K.-N., An introduction to atmospheric radiation, (Academic Press, New York, 1980, pp. 223).

Liu Q., C.Simmer, and E.Ruprecht, "3-D Radiation Transfer Effects of Clouds in the Microwave Spectral Range", J.Geophys. Res., 101, D2, 4289-4298, 1996. 
Petty, G. W., "Physical retrievals of over-ocean rain rate from multichannel microwave imagery. Pert I: Theoretical characteristics of normalized polarization and scattering indeces", Meteorol. Atmos. Phys., 54, 79-99, 1994.

Roberti L., J.Haferman, and K.Kummerow, "Microwave radiative transfer through horizontally inhomogeneous precipitating clouds,"J. of Geoph. Res., 99, 16, 707-16,718, 1994.

Roberti L., C. Kummerow, "Monte Carlo calculations of polarized microwave radiation emerging from 3-D cloud structures", Proceeding IGARSS'95, Florence, Italy, 708-710, 1995.

Roberti L., "Monte Carlo radiative transfer in the microwave and in the visible: biasing techniques.", Applied Optics, 36, 7929-7938, 1997.

Tao K., J.Simpson and S.-T. Soong, "Statistical Properties of a Cloud Ensemble: A Numerical Study," J. of Atm. Sci., 44, 3175-3187, 1987.

Tripoli G. J., A nonhydrostatic model designed to simulate scale interactions, Mon. Weather Rev., 120, 1342-1359, 1992.

Tsang T., J.A. Kong, R.T. Shin, Theory of microwave remote sensing, (John Wiley and Sons, New York, 1985), Chap. 3, pp. 138-142. 
Tsang T., J.A. Kong, R.T. Shin, Theory of microwave remote sensing, (John Wiley and Sons, New York, 1985), Chap. 3, pp. 138-142.

Weinman J.A., and P.J. Guetter, Determination of rainfall distributions from microwave radiation measured by the Nimbus 6 ESMR,, J. Appl. Meteorol., 16, 437-442, 1977.

Weinman J.A., and R. Davis, Thermal microwave radiances from horizontally finite clouds of hydrometeors, J. Geophys. Res., 83, 3099-3107, 1978. 


\section{FIGURE CAPTIONS}

Figure 1: $T_{B}$ for a subcloud in the anvil region and one in the core region of the cloud for density of snow $0.1 \mathrm{~g} / \mathrm{cm}^{3}, 3$ different frequencies $(19.35,37.0,85.6 \mathrm{GHz}$.), obtained with the non polarised backward Monte Carlo code ( $\_$)and the forward code (---).

Figure 2: $T_{H}$ and $\Delta T_{V-H}$ for a subcloud in the stratiform region of the cloud for density of snow $0.1 \mathrm{~g} / \mathrm{cm}^{3}, 3$ different frequencies $(19.35,37.0,85.6 \mathrm{GHz}$.$) , obtained with the polarized$ backward-forward Monte Carlo code ( $\_$), the forward code for spherical particles (-- ) and the forward code for oriented spheroidal particles $(\cdots)$.

Figure 3: $\Delta T_{V-H}$ for a subcloud in the stratiform region of the cloud for density of snow 0.1 and $0.2 \mathrm{~g} / \mathrm{cm}^{3}$ and 5 percentages of graupel transformed to snow $(0,25,50,75$ and $100 \%)$. 19.35 $\mathrm{GHz},(---) 37.0 \mathrm{GHz},(\cdot) 85.6 \mathrm{GHz}$.

Figure 4: $\Delta T_{V-H}$ for a subcloud in the convective region of the cloud for density of snow 0.1 and $0.2 \mathrm{~g} / \mathrm{cm}^{3}$ and 5 percentages of graupel transformed to snow $(0,25,50,75$ and $100 \%)$. ) $19.35 \mathrm{GHz},(---) 37.0 \mathrm{GHz},(\cdots) 85.6 \mathrm{GHz}$.

Figure 5: $T_{H}$ and $\Delta T_{V-H}$ associated to the considered cloud section. The density of snow is $0.1 \mathrm{~g} / \mathrm{cm}^{3}$, the asimmetry parameter $\mathrm{a} / \mathrm{b}$ is $0.3 .(19.35 \mathrm{GHz},(--) 37.0 \mathrm{GHz},(\cdots) 85.6$ $\mathrm{GHz}$ 

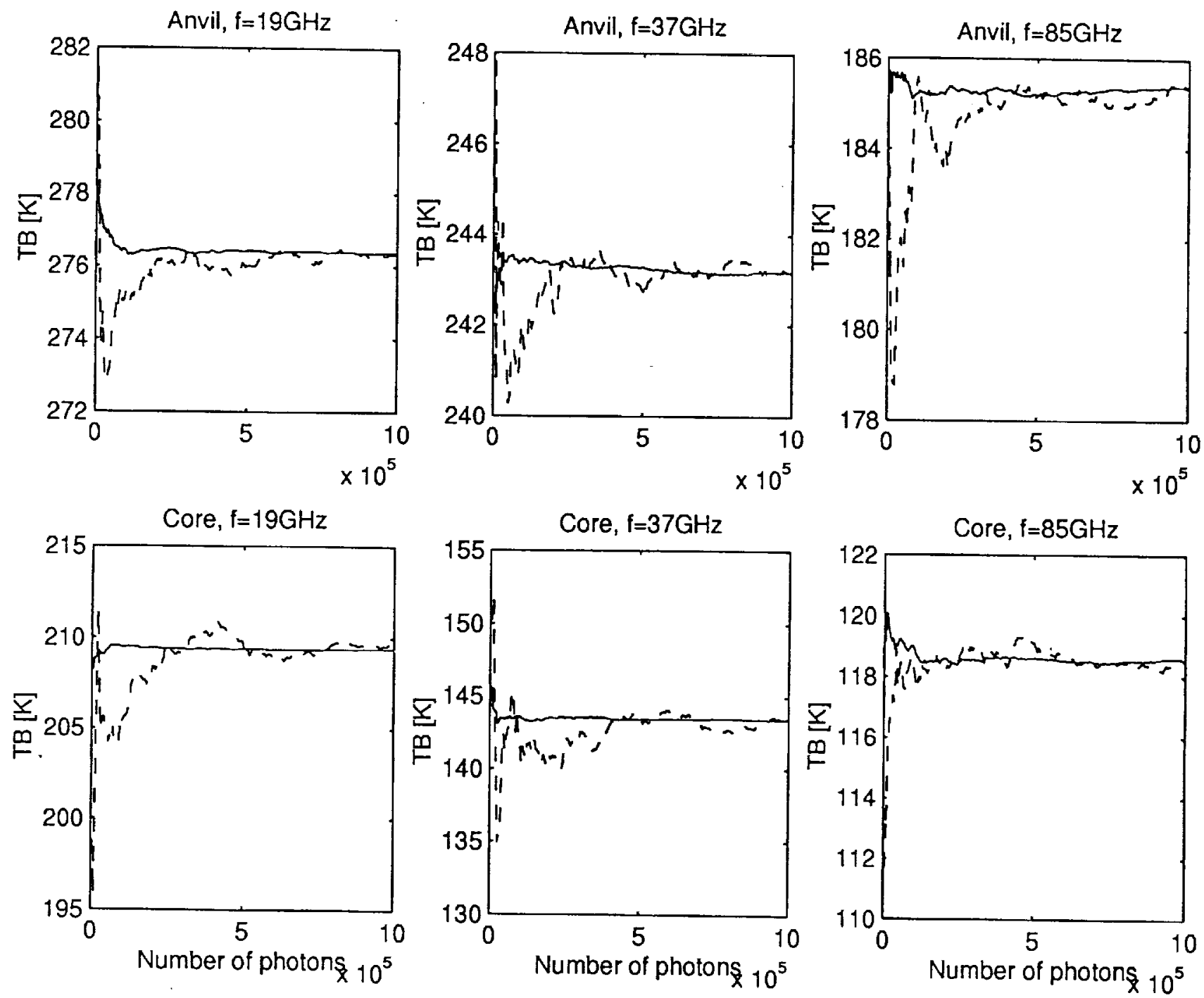

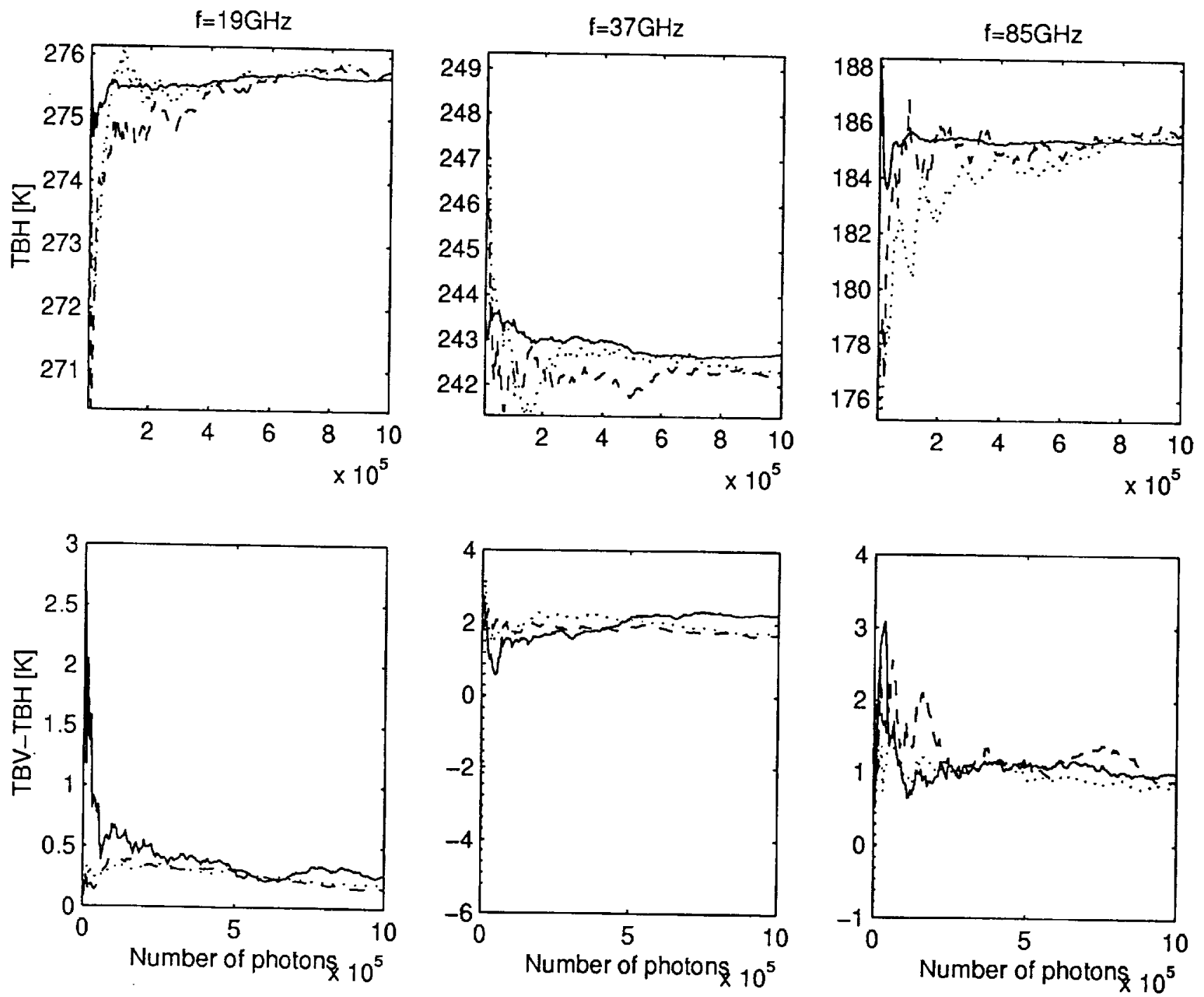
Graup.-snow 0\% Graup.-snow 25\% Graup.-snow 50\% Graup.-snow 75\% Graup.-snow 100\%
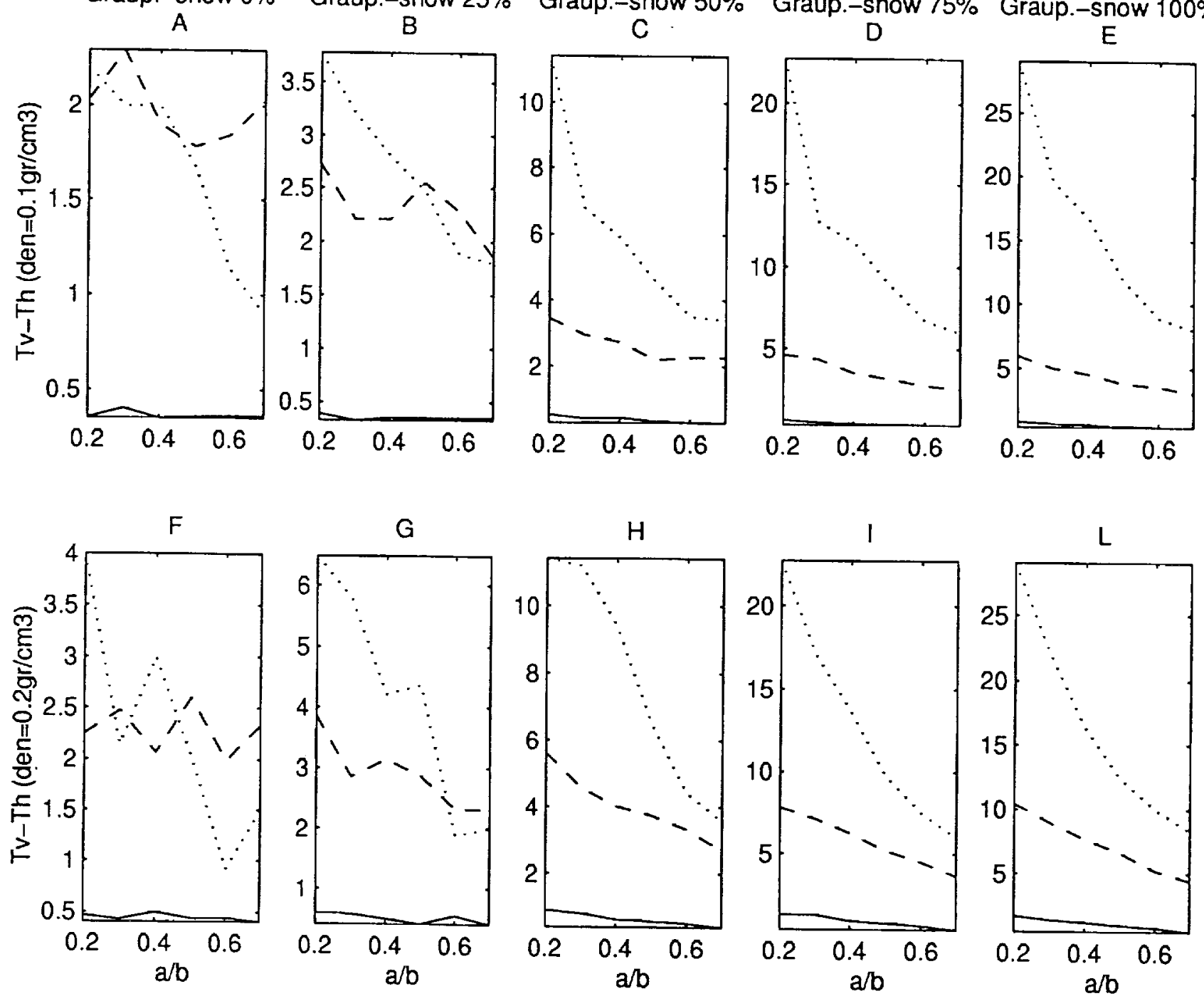


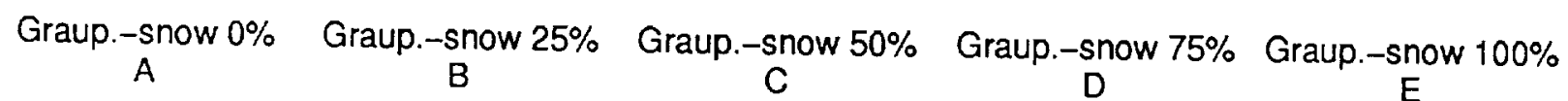
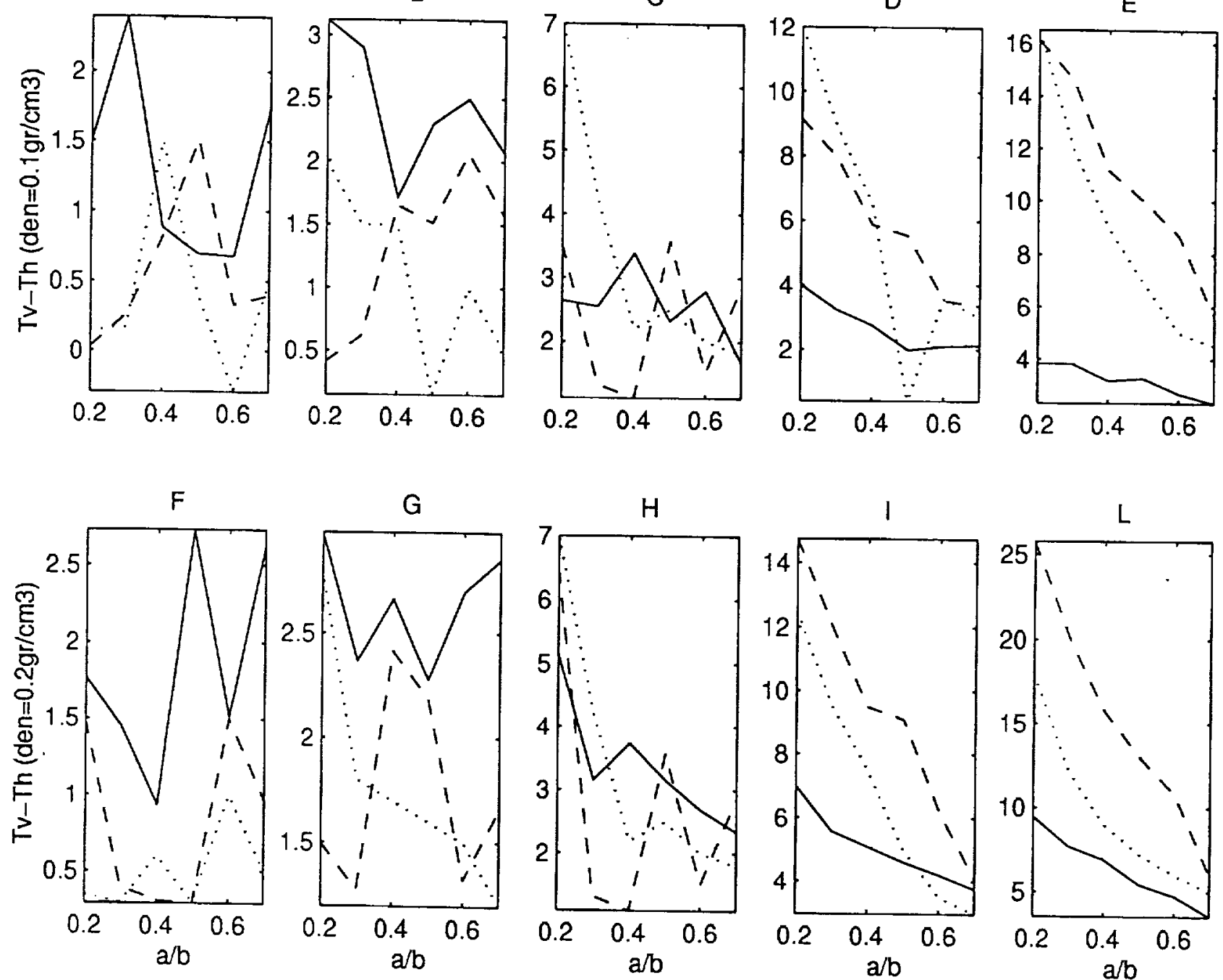

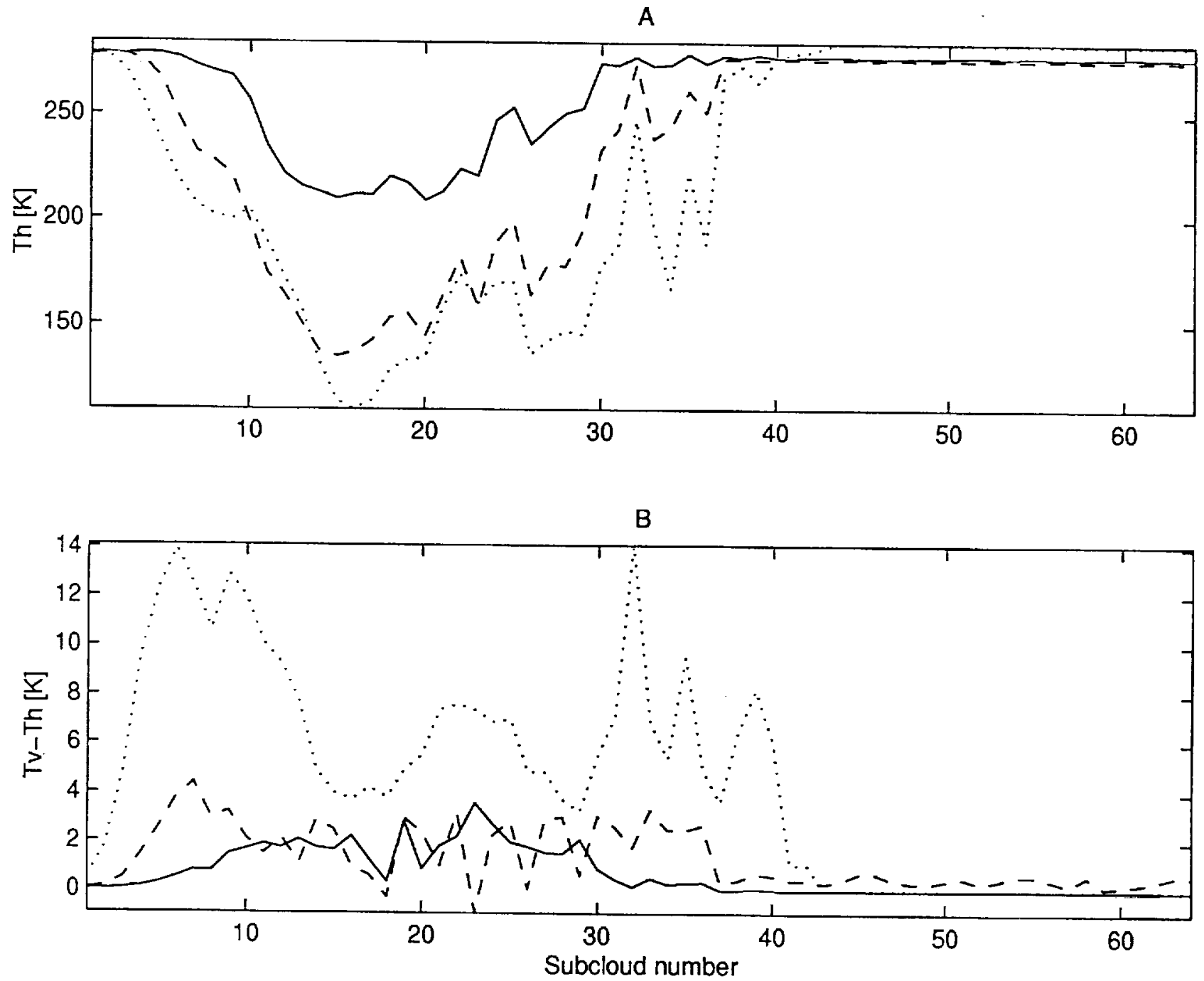